\title{
UV-LIGA Microfabrication Process for sub-Terahertz Waveguides Utilizing Multiple Layered SU-8 Photoresist
}

\author{
Ali Malekabadi, Claudio Paoloni \\ Engineering Department \\ Lancaster University, \\ Lancaster, LA1 4YW, UK \\ E-mail: c.paoloni@lancaster.ac.uk
}

\begin{abstract}
.
A microfabrication process based on UV LIGA (German acronym of lithography, electroplating and molding) is proposed for the fabrication of relatively high aspect ratio sub-terahertz $(100-1000 \mathrm{GHz})$ metal waveguides, to be used as slow wave structure (SWS) in sub-THz vacuum electron devices. The high accuracy and tight tolerances required to properly support frequencies in the sub-THz range can be only achieved by a stable process with full parameter control. The proposed process, based on SU-8 photoresist, has been developed to satisfy high planar surface requirements for metal sub-THz waveguides. It will be demonstrated that, for a given thickness, it is more effective the stacking of a number of layers of SU-8 with lower thickness rather than using a single thick layer obtained at lower spin rate. The multiple layer approach provides the planarity and the surface quality required for electroforming of ground planes or assembly surfaces and for assuring low ohmic losses of waveguides. A systematic procedure is provided to calculate soft and post-bake times to produce high homogeneity SU-8 multiple layer coating as mold for very high quality metal waveguides. A Double Corrugated Waveguide (DCW) designed for $0.3 \mathrm{THz}$ operating frequency, to be used in vacuum electronic devices, was fabricated as test structure.

The proposed process based on UV LIGA will enable low cost production of high accuracy sub-THz threedimensional waveguides. This is fundamental for producing a new generation of affordable sub- $\mathrm{THz}$ vacuum electron devices, to fill the technological gap that still prevents a wide diffusion of numerous applications based on $\mathrm{THz}$ radiation.
\end{abstract}

Index Terms-UV-LIGA, microfabrication, vacuum electron device (VED), double corrugated waveguide (DCW), multi-layer coating, terahertz (THz), SU-8, millimeter waves 


\section{Introduction}

The recent progresses in microfabrication are enabling a new generation of vacuum electronic devices (VEDs) at sub-terahertz (sub-THz) frequencies $(100-1000 \mathrm{GHz})$ to generate output power at Watt level [1 - 9]. The solid state technology, limited to the milliwatt range, is not able to satisfy the power needs in this portion of the spectrum. The availability of relatively high power compact sub$\mathrm{THz}$ sources is pivotal for a large number of applications in fundamental fields as communications, healthcare, imaging $[10,11]$. The metal structures to provide adequate electromagnetic performance in the sub-THz range, due to challenging small dimensions, have to be built with high accuracy and surface finishing [4]. In particular, the Ohmic losses due to a poor surface roughness could strongly affect the overall performance. The surface roughness has to be maintained substantially lower than the skin depth, typically on the order of tens of nanometers. Further, depending on the structure geometry, wide high planarity metal surfaces are required.

Different microfabrication processes are used for the realization of VEDs for the sub-THz region [2 - 9], such as photolithographic processes and high precision CNC milling. The CNC milling was proved effective up to 0.35 $\mathrm{THz}$ [12] by a number of prototypes. At higher frequencies the smaller dimensions are achievable only by a photolithographic approach. Two photolithographic techniques are mostly used to built high aspect ratio three dimensional structures, the Deep Reactive Ion Etching (DRIE) $[9-13,15]$ and the LIGA [2 - 8].

The DRIE method is based on etching a silicon substrate and then coating it with a metal layer. A metal-coated structure provides very good electrical characteristics, but not very good thermal dissipation.

LIGA is a process of high flexibility based on the exposure of thick photoresist to a specific light source, to build relatively high aspect ratio three dimensional mold with an arbitrary 2D pattern [16] for electroforming of metal micro structures, typically copper. This process, in principle, provides an excellent accuracy, repeatability and low tolerances, however, it has to be properly calibrated on the basis of the geometry of the structure considered to achieve optimum results. Deep X-ray LIGA is an expensive process, based on a Synchrotron light source and PMMA resist [5], to produce high aspect-ratio up to 50-100:1. UV LIGA is an affordable process based on a conventional UV light source to expose resists as SU-8, an epoxy-based photoresist, or KMPR [6]-[8]. It is suitable for moderate aspect-ratio (up to about 20:1).

The KMPR resist [17] is of easy removability, but has a higher sensitivity to UV exposure and a lower mold quality compared to SU-8. The SU-8 photoresist is preferred for the fabrication of sub-THz structures due to its excellent resolution and consistently high quality molding [2]. The removal of SU-8 is very difficult, especially if low surface roughness metal surfaces have to be obtained.

UV-LIGA can be applied to different substrates such as silicon, copper or quartz. In case of copper substrates, an initial polishing step is needed to avoid the scattering of UV light from the metal surface.

One of the main challenges of UV-LIGA is to build perfectly flat metal surfaces as a ground plane or as bonding surface, e.g, for the assembly of two parts of a complex structure [7]. A high precision control of the SU8 thickness and planarity has to be achieved to avoid lapping and polishing steps and for a high repeatability at low cost [4].

The UV-LIGA SU-8 process was demonstrated effective for the fabrication, e. g., of a $220 \mathrm{GHz}$ sheet beam Travelling-Wave Tube (TWT) slow wave structure [6] or $140 \mathrm{GHz}$ folded waveguide [7].

In this paper, an advanced approach for UV LIGA, based on multiple layers of SU-8, to produce metal waveguides for sub-THz frequency range, is proposed.

The effectiveness of the process is demonstrated by the fabrication of a test waveguide, the double corrugated waveguide (DCW) [18], to be used as slow wave structure (SWS) in a Backward Wave Oscillators (BWOs) [19] at $0.3 \mathrm{THz}$. The BWO is a vacuum electron device suitable to provide high power in the $\mathrm{THz}$ frequency range. The core of the BWO is a waveguide, the DCW in this case, shaped to permit the transfer of energy from a high energy electron beam, magnetically confined, flowing in a channel along the longitudinal direction, to the radiofrequency field. The electron beam is generated by an electron gun and is collected at the end of the SWS by a collector to increase the efficiency.

In the following, the UV-LIGA process based on a multiple-layer SU-8 coating for accurate fabrication of sub-THz waveguides will be described.

\section{Fabrication of slow wave structures for sub-THz vacuum electron devices}

Typically, sub-THz SWSs are derived from the family of corrugated waveguides. They have a moderate aspect ratio and period length as a function of the operating frequency [5]. Their geometry is chosen to be fabricated by photolithography with a defined $2 \mathrm{D}$ pattern printed on a mask, while the height of the corrugations is set by the resist thickness. The double corrugated waveguide (Fig. 1) has been proved to be a very promising SWS [18] from microwaves to $1-\mathrm{THz}$. It was realized for the $1 \mathrm{THz}$ 
frequency range by Deep X-ray LIGA [5] and at 0.346 $\mathrm{THz}$ by nano-CNC milling [12]. The latter technique is very effective for prototyping. The DCW consists of two parallel rows of pillars of given height and cross section, equally spaced in a periodic pattern (Fig. 1). The fabrication of the pillar of the DCW presents two main challenges. One is the small size and the relative short mutual distance, the second is the high accuracy to produce the same height for all the pillars, to properly support the given operating frequency.

The aspect ratio of the structure in the $3: 1$ to $5: 1$ range (Fig. 1b) makes UV LIGA an effective and affordable microfabrication technique. However, the shape of the DCW requires an ad-hoc process to achieve the required specifications.

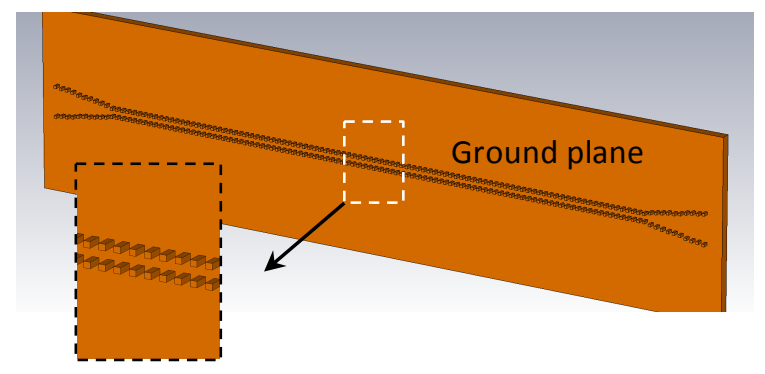

a)

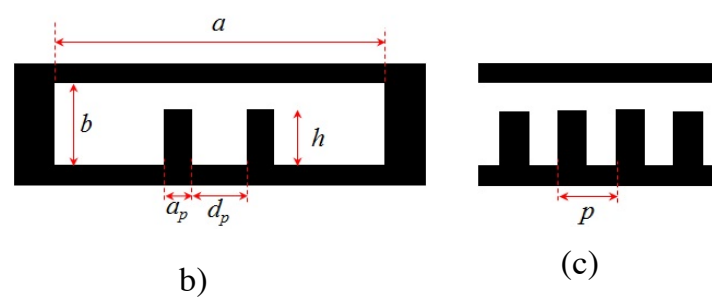

Fig. 1. Double corrugated waveguide full structure (a). The pillars are connected on a wide ground metal plane. Cross section (b) and longitudinal section (c).

Two approaches can be considered for the fabrication by UV-LIGA. One approach is to grow the pillars on a ground plane of copper coated by SU-8. The height of the pillar is defined by a precise control of the electroforming process that has to be stopped when the copper reaches the SU-8 surface. This approach is quite demanding and requires a lapping step to assure a smooth surface at the top of the pillars. The second approach is based on the growing of pillars from their top toward the ground plane, by using a metallized silicon wafer coated by SU-8 as substrate. The advantage of this approach is that the height of the pillars is precisely defined by the SU-8 thickness. After the pillars have reached the SU-8 surface,

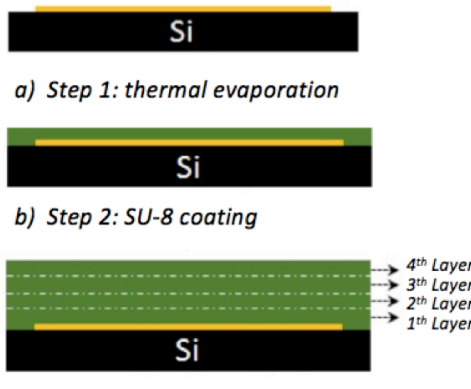

c) Step 3: SU-8 coating successive layers

Fig. 2. The first three steps of the multiple layer UV LIGA. A seed layer is evaporated on the $\mathrm{Si}$ wafer (a). The wafer is then coated (b) with the number of layers required for the given thickness (c).

the electroforming process continues for growing a robust metal ground plane.

Two other important specifications are tolerances in the range of a few microns and surface roughness better than $100 \mathrm{~nm}$.

\section{UV LIGA for sub-THz slow wave structures}

The process described in the following is applied to the fabrication of a $0.3 \mathrm{THz} \mathrm{DCW}$, but it is suitable to build any three dimensional waveguide based on a $2 \mathrm{D}$ pattern, in a wide range of dimensions as a function of the operating frequency. The DCW dimensions for $0.3 \mathrm{THz}$ operating frequency are $a=1500 \mu \mathrm{m}, b=200 \mu \mathrm{m}, h=$ $160 \mu \mathrm{m}, d_{p}=120 \mu \mathrm{m}, p=160$ and $a_{p}=70 \mu \mathrm{m}$ (Fig. 1). The SU-8 2075 photoresist has been chosen as the best compromise between viscosity and achievable thickness [20].

While the mask provides very accurate dimensions for the 2D pattern, a correct height of the pillars requires a high precision SU-8 thickness. The approach, based on coating of a silicon wafer to grow the copper starting from the top of the pillar, benefits from the high planarity of the silicon wafer, avoiding the need of lapping of the top of the pillars.

The area of the $0.3 \mathrm{THz}$ DCW SWS (Fig.1) is in the range 1-2 $\mathrm{cm}^{2}$. A 2-inch wafer can accommodate 4-5 structures. The use of large wafer could be considered in case of production of large number of samples.

The application of the SU-8 on the wafer is a well established and apparently simple procedure. However, in reality, the distribution of the SU-8 presents intrinsic difficulties to achieve a high homogeneity and planarity. The quality of the SU- 8 surface determines the metal surface characteristics of the ground plane of the DCW. Any defect on the SU-8 surfaces could affect the quality of the metal structure. Typically, at the increase of the thickness of a SU-8 single layer, the presence of defects increases. 

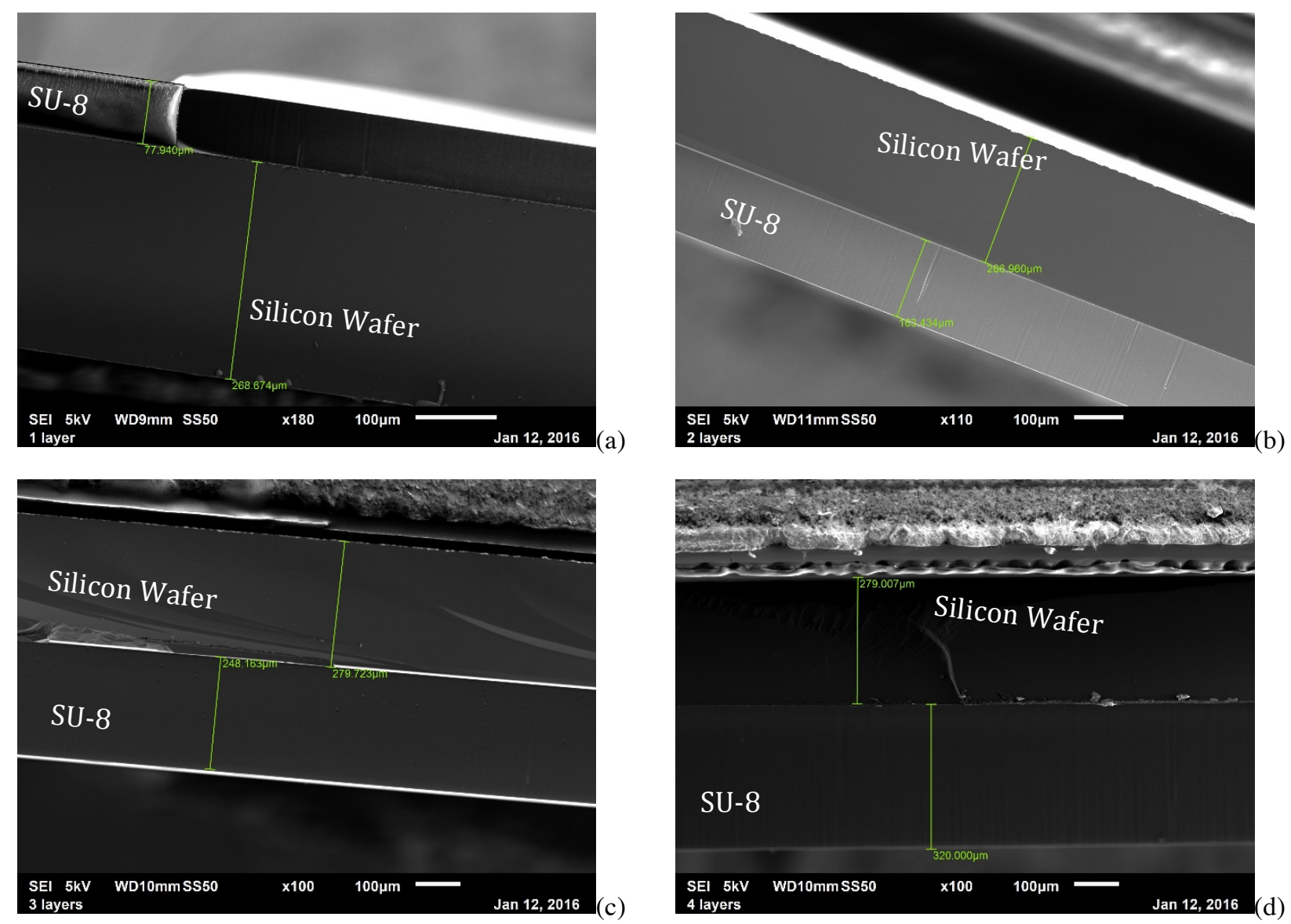

Fig. 3. Thickness of SU-8 (a) $h_{l}=78 \mu \mathrm{m}$, one layer coating, (b) $h=163 \mu \mathrm{m}, n=2$, two layer coating, (c) $h=248 \mu \mathrm{m}, n=3$ three layer coating, and (d) $h=320 \mu \mathrm{m}, n=4$, four layer coating. The silicon wafer has $270 \pm 10 \mu \mathrm{m}$ thickness.

The proposed approach will solve the issue by using a multiple layer SU-8 process. Once the process parameters for a thin layer are well established for a given thickness, a number $n$ of layers can be stacked to reach a final thickness, as an integer multiple of the initially defined single layer thickness. This produces a relevant improvement of the overall time of setting of the process parameters. It will be shown that at the increase of the number of staked layers the quality of the SU-8 surface improves substantially, with practically no defects in comparison to the single layer approach.

The thickness of the coating is a function of the viscosity and the spinning speed. It is well known that beads form at the edge of the wafer with height and extensions inverse function of the speed, due to discontinuous shear forces. SU-8 2075 photoresist provides a very uniform layer and low beads when coated at spin speed higher than $2000 \mathrm{rpm}$, for thickness up to $100 \mu \mathrm{m}$. Thicker layers can be obtained by decreasing the spin rate below 1500 $2000 \mathrm{rpm}$, but this produces high edge beads of excess photoresist, critical for the wafer-mask contact. High beads tend to flow toward the center of the wafer drastically decreasing the usable area and are difficult to remove. Beads can be removed manually or automatically depending on the spin coater used. The multiple layer coating technique substantially alleviates the above discussed issues.

\subsection{SU-8 deposition and development}

The first step of the multiple layer UV LIGA process is the metallization of the silicon wafer with $20 \mathrm{~nm}$ of titanium (Ti) followed by thermal evaporation of $175 \mathrm{~nm}$ of nickel (Ni) (Fig.2a). Nickel is chosen as seed layer due to its poor adhesion with copper, to facilitate the separation of the final electroformed metal structure from the silicon wafer. The Ti layer improves the adhesion between the seed layer $(\mathrm{Ni})$ and surface of the silicon wafer. The seed layer works also as electrode for the electroforming.

The second step is the coating of SU-8 (Fig.2b). A thickness in the order of few tens of microns can be easily achieved by a single coating. In the case of mediumviscosity photoresist such as SU-8 2075, and for thicknesses of more than $150 \mu \mathrm{m}$ for a single coating, a great effort is required a for the precise tuning of process parameters (spin speed, soft baking) to avoid wavy and uneven depositions.

Given $h$ the total thickness of the SU-8 coating required to provide the correct pillar height, instead of using a single layer of thickness $h$, a number $n$ of layers with thickness 


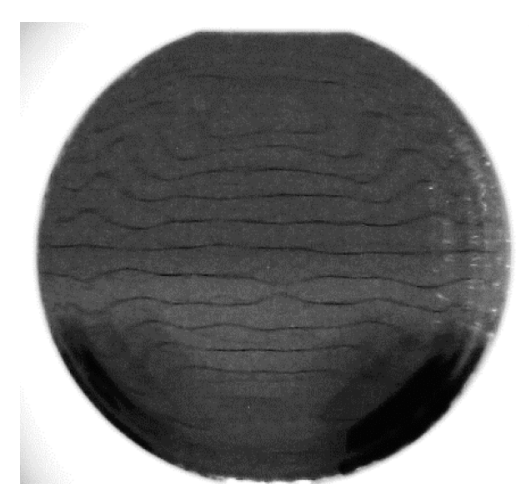

a)

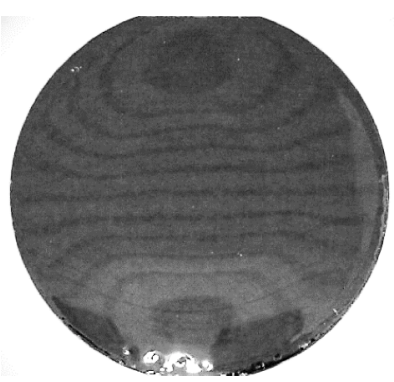

b)

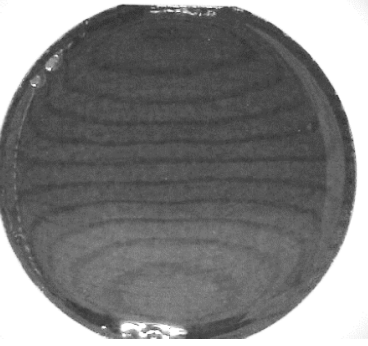

c)
Fig. 4. SU-8 coated 2-inch Silicon wafers (a) one layer (b) two layers and (c) three layers. Due to self-planarization properties of the SU8, the quality of the surface improves as the number of layer increase. The lines are just reflection of the light from a notebook paper to clarify the defects.

$h_{l}=h / n$, where $h_{l}<100 \mu \mathrm{m}$ is achievable with a high spin rate of about $2300 \mathrm{rpm}$, is used (Fig. 2c). In case of the $0.3 \mathrm{THz}$ DCW, the thickness required (Fig. 1) is $h=$ $160 \mu \mathrm{m}$. In the following, a single layer with $h_{l}=80 \mu \mathrm{m}$ is considered. A Spinner Suss Microtec LabSpin 6 was used for the purpose.

Once the process parameters for the single layer coating are defined, any thickness multiple integer of $h_{l}=80 \mu \mathrm{m}$ can be obtained without recalibration. Fig. 3 shows the SU-8 coating for one (a), two (b), three (c) and four (d) layers on 2" Silicon wafer with $270 \pm 10 \mu \mathrm{m}$ thickness, to achieve $h=320 \mu \mathrm{m}$. The first layer has a thickness $h_{l}=$ $78 \mu \mathrm{m}(\Delta=-2.5 \%)$, the two layer coating $h=163 \mu \mathrm{m}(\Delta$ $=+1.18 \%)$, the three layer coating $h=248 \mu \mathrm{m}(\Delta=+3.3$ $\%)$ and finally the four layer coating provide the required $h=320 \mu \mathrm{m}(\Delta=0 \%)$. The maximum tolerance is always below the $3.3 \%$ in the intermediate layers, while practically $0 \%$ after the fourth layer.

To note, that according to the SU-8 2075 data sheet, 2300 rpm gives around $90 \mu \mathrm{m}$ of thickness instead of the obtained $80 \mu \mathrm{m}$. It confirms how the different environmental conditions in the clean room can alter the data sheet values.

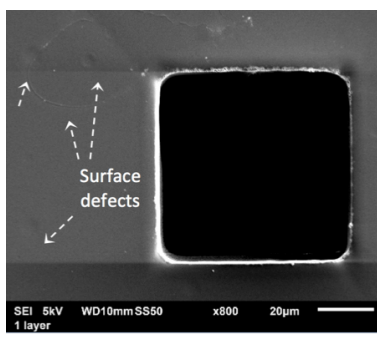

a)

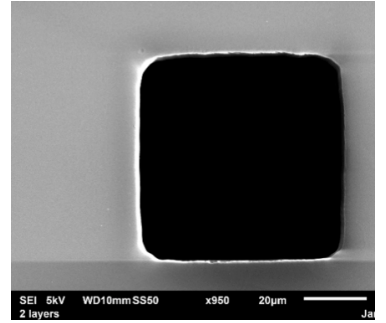

b)
Fig. 5. Comparison between the surface quality of samples with one layer (a) of SU-8 and with 2 layers (b) of SU-8. The thickness of resist in (a) is $80 \mu \mathrm{m}$ while in (b) is $160 \mu \mathrm{m}$.

High planarity SU-8 surfaces are a requirement to grow by electroforming wide perfectly flat metal surfaces as ground planes or assembly surfaces for waveguides. The microscopic images of the SU-8 surface for different number of layers are shown in Fig. 4. To evidence the presence of planarity defects, the samples were illuminated by a light reflected from a wide ruled notebook sheet. In case of one layer (Fig. 4a), one can observe a very irregular pattern that demonstrates the presence of bumps and defects on the SU-8 surface. After the second layer is coated, the bumps are substantially reduced (Fig. 4b). In case of three layers (Fig. 4c), it is notable a regular pattern showing the high homogeneity of the surface with respect to the single layer. Fig. 5 shows the detail in proximity of a $70 \mu \mathrm{m}$ hole of the SU-8 surface quality after UV exposure. The defects, visible in case of one layer (Fig. 5a), disappear in the two layer sample (Fig. 5b).

Moreover, the multiple layer technique permits to obtain a more precise control of the resist thickness in comparison to the single layer technique.

A systematic approach is described to calculate the process parameters as function of the number of layers required for a given SU-8 thickness $h$ of the sample.

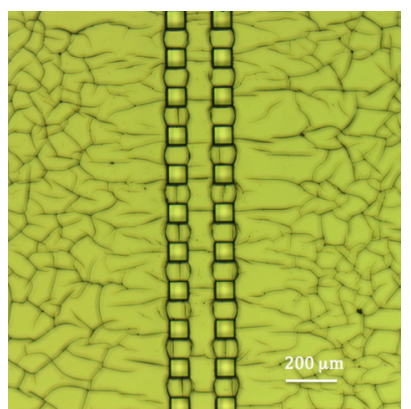

a)

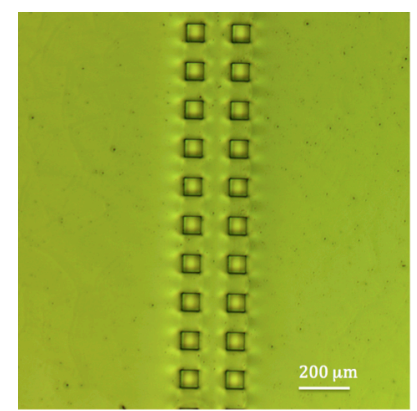

b)
Fig. 6. Effect of I-line filter (a) crack appeared during the development of the resist, (b) no crack after post bake annealing. 
Once the process is set for one layer, the parameter to modify at the increase of the number of layers is the soft bake time according to the procedure below

$$
\text { Soft bake time: } \mid \begin{aligned}
& \text { at } 65^{\circ} \mathrm{C}: 5+(n-1)[\mathrm{min}] \\
& \text { at } 95^{\circ} \mathrm{C}: 15+(n-1)[\mathrm{min}] \\
& \text { Relaxing time : } 10[\mathrm{~min}]
\end{aligned}
$$

where $n$ is the $\mathrm{n}^{\text {th }}$ SU- 8 layer. After the soft-bake, the sample has to be relaxed for another 10 minutes. This will make the coated SU-8 more robust to support the pouring of the next layer, without affecting the surface quality. The hotplate has to be perfectly horizontal to avoid an uneven distribution of the SU-8 during the soft-backing process.

The MJB4 mask aligner (from SUSS Microtec) is used for the alignment and exposure, with the I-line filter. The optimum exposure energy as a function of the number of layer has been found to be:

Exposure energy: $\mid \begin{array}{ll}300 \times n & \mathrm{~mJ} / \mathrm{cm}^{2}-\text { with I-line filter } \\ 200 \times n & \mathrm{~mJ} / \mathrm{cm}^{2}-\text { without I-line filter }\end{array}$ where $n$ is the $n^{\text {th }}$ coated layer. As expected, at the increase of the number of staked layers, higher energy is required. The I-line filter substantially improves the quality of borders. This is very important in sub-THz waveguides where small fluctuations in the surface of the waveguide results in high losses [11]. The disadvantage of using I-line filter is in the cracks that appear on the SU8 surface (Fig. 6a) during the development, even if the exposure density is increased by about $40 \%$ as suggested by the datasheet. Although hard baking can anneal the resist, it is not a viable solution since it melts and can cause resist replacement. In addition, hard baking needs a very long cooling down process otherwise, due to the tensions inside the resist and low adhesion of SU-8 to the surface of silicon wafer, the SU-8 can detach or even break the wafer.

After UV exposure, the Post-Exposure Baking (PEB) was found the best solution to remove cracks (Fig. 6b). The optimum PEB is the same of the soft-bake:

Post-exposure bake time: $\mid \begin{aligned} & \text { at } 65^{\circ} \mathrm{C}: 5+(n-1)[\mathrm{min}] \\ & \text { at } 95^{\circ} \mathrm{C}: 15+5 \times(n-1)[\mathrm{min}] \\ & \text { Relaxing time :10 [min] }\end{aligned}$

where $\mathrm{n}$ is refer to the $n^{\text {th }}$ layer. To avoid cracks in the resist, the sample is ramped slowly to the room temperature. Cooling down too quickly the temperature of the wafer may result in separation of the resist from the silicon substrate.

The process continues by $8 \times n$ minutes of development of the SU-8 in a gentle ultrasonic agitation. A

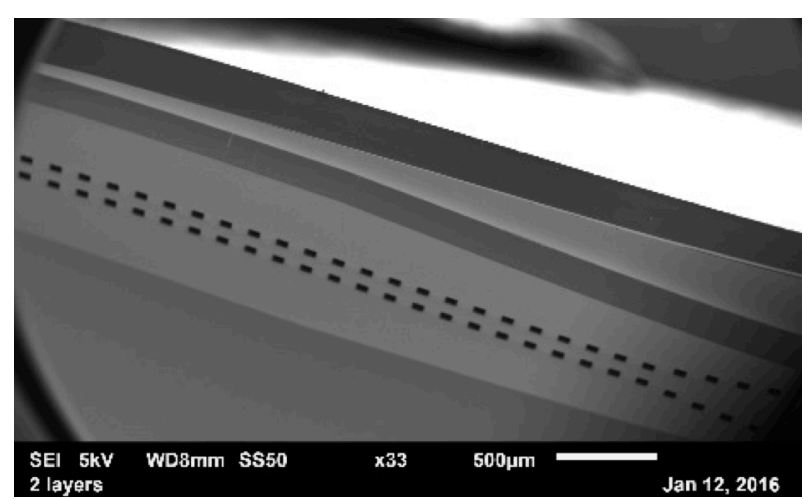

a)

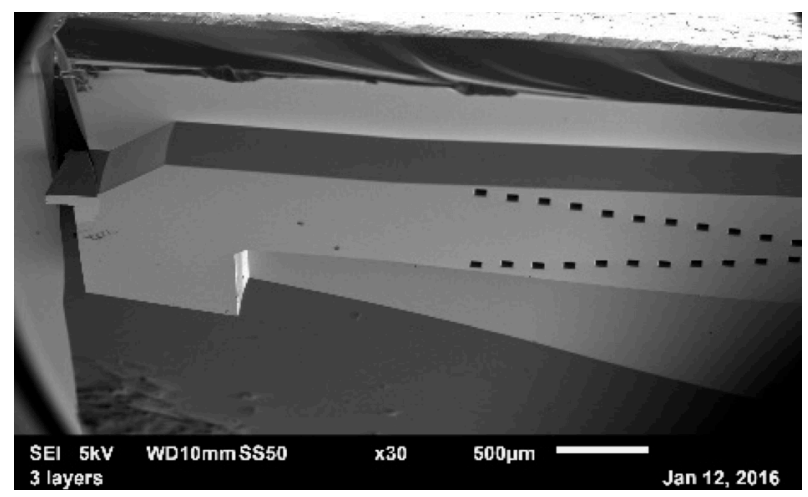

b)

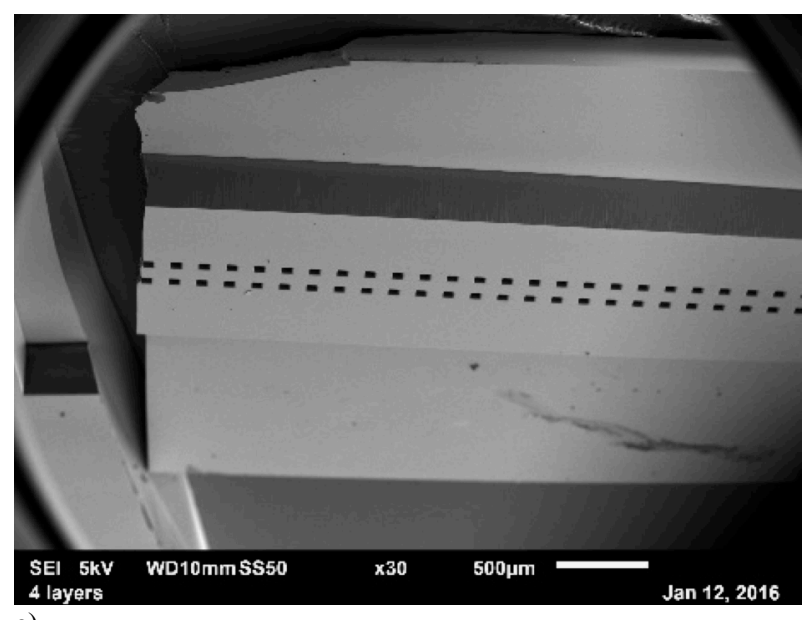

c)

Fig. 7. SEM photos of the samples after lithography: (a) two layers, (b) three layers and (c) four layers.

commercially available SU- 8 developer is used for the development. To remove any SU-8 residual, the developed sample is placed into the plasma chamber for 10 minutes for Oxygen plasma cleaning. Fig. 7 shows the SEM pictures of the samples after the lithography step for 2 (Fig. 7a), 3 (Fig. 7b) and 4 layers (Fig. 7c) of SU-8. It is notable the high quality of the surfaces. The samples were tilted to have a good photo perspective. 

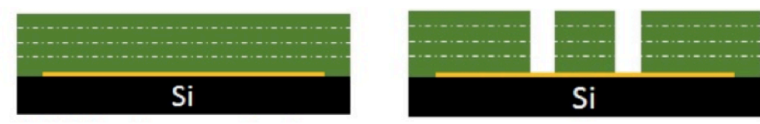

a) Step 3: SU-8 coating successive layers b) Step 4: exposure and development

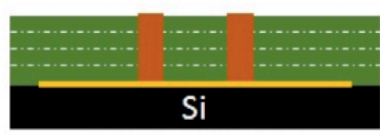

c) Step 5: electroforming

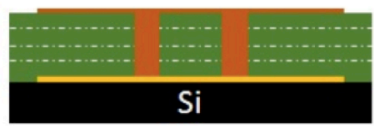

d) Step 6: evaporation
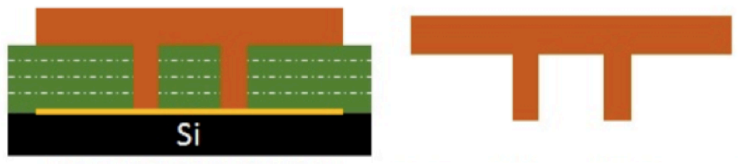

e) Step 7: second electroforming

Fig. 8. Steps 3 to 8 of DCW fabrication process. After exposure and development (b), the copper structure is built (c) by electroforming. A second evaporation (d) follows and a solid ground plane (e) is growth. The metal structure is then detached from the silicon wafer and SU-8 is removed by a RIE (f).

Fig. 8 summarizes the fabrication steps after the SU-8 coating. The sample is masked and exposed to the UV light (Fig. 8b) in the mask aligner, and developed. Then the sample is placed in the electroforming bath. As soon as the copper reaches the surface of the SU-8 (Fig. 8c), the sample is removed from the bath and a second metal deposition of $200 \mathrm{~nm}$ (Fig. 8d) by thermal evaporation is performed to create the seed layer for the ground plane. The sample is immersed in the bath for a second electroforming step to build a robust ground plane with a thickness in the range of 1-2 mm (Fig. 8e). Finally, the sample is removed from the bath, the silicon wafer is detached from the metal structure embedded in the SU-8 and the SU-8 is removed from the metal structure (Fig. 8f).

\subsection{Copper Electroforming}

In order to avoid the electroforming of copper on unwanted areas, especially on the edges of the wafer, where the current is more intense, the seed layer is only coated at the center of the wafer where the SU- 8 will be masked (Fig. 1). This prevents the growth of large amount of copper on the edges and will help to remove the SU-8 more easily. The electroforming solution consists of Sulfuric acid $\left(\mathrm{H}_{2} \mathrm{SO}_{4}\right) 190 \mathrm{~g} / \mathrm{l}$ [2]. The main requirement is a very low surface roughness, in the range of $50-100$ $\mathrm{nm}$, to have a low losses metal waveguide. A DC current density of $5 \mathrm{~mA} / \mathrm{cm}^{2}$ was found suitable to produce very fine copper grain size. A magnetic stirrer is used for the agitation of the solution.

The electroforming of pillar height $h=160 \mu \mathrm{m}$ needs about 48 hours. The time to grow a $2 \mathrm{~mm}$ thick ground plane is about one week.
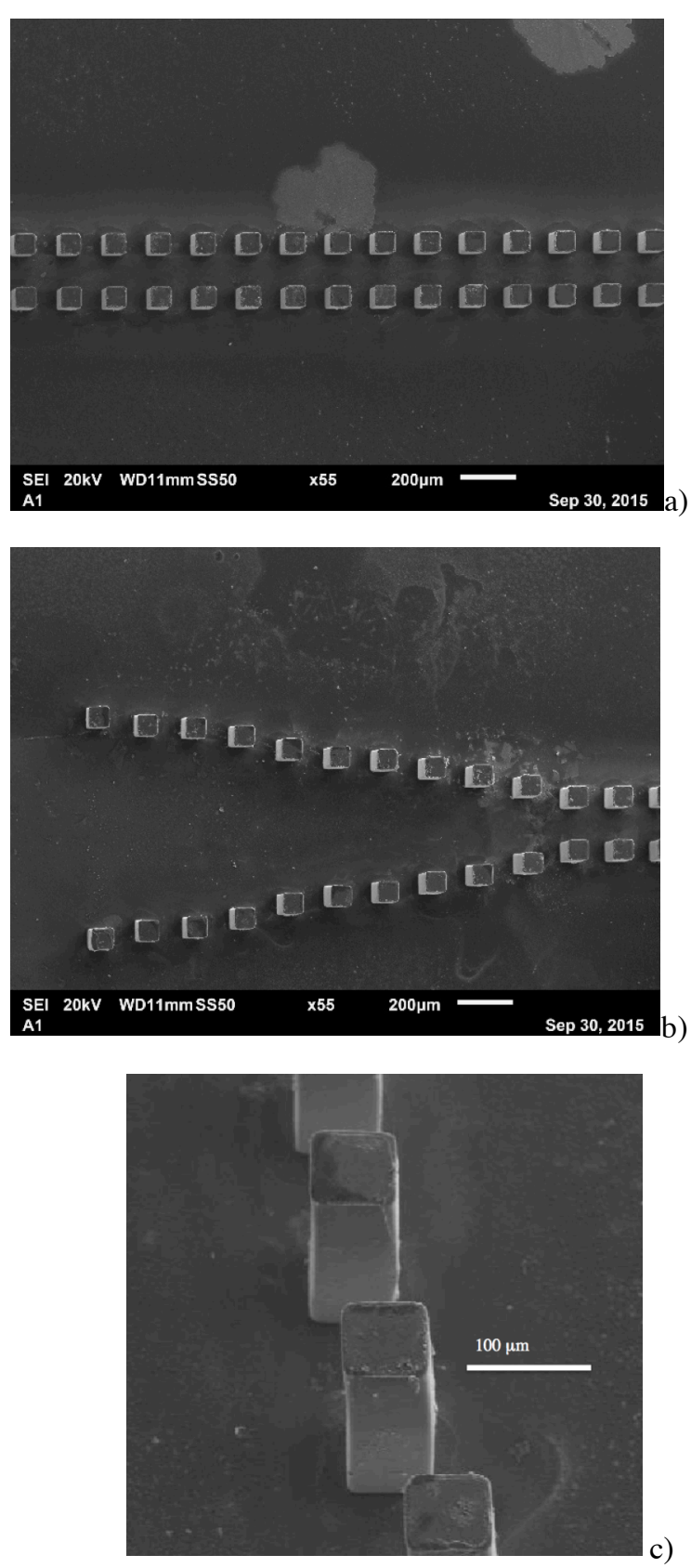

Fig. 9 Details of the copper DCW after the SU-8 removal (a) middle section, b) coupler, c) pillars)

\section{$3.3 S U-8$ removal from the metal structure}

At the end of the electroforming process, the silicon has to be separated from the Copper/SU-8 structure. This phase is quite critical due to the risk of damaging the small pillars [21 - 23]. Initially, the sample is placed inside a Reactive Ion Etching (RIE) machine for a plasma etching process for one hour. A gas mixture of Oxygen $\left(\mathrm{O}_{2}\right)$ and $\mathrm{CF} 4$ at the rate of $50 \mathrm{sccm}$ and $5 \mathrm{sccm}$ respectively is used. The RIE machine, Oxford Instruments PlasmaPro NGP80, is set to provide $200 \mathrm{~W}$ at $100 \mathrm{mT}$ of pressure. Once the plasma etching is performed, the silicon wafer can be safely detached from the structure comprising the SU-8 and the copper by applying a very weak shock. The second step is to remove 
the SU-8 from the copper structure. It is well known how difficult this phase is and numerous methods are proposed for the purpose [2]. This is particularly demanding in the regions between pillars, where very narrow spaces make arduous the removal. It has been found that very good results are obtained by a second phase of the RIE for 4 hours.

Fig. 9 shows details of the final copper DCW after the SU-8 removal. It is noteworthy the high quality of the surfaces and the very good definition of the geometry.

\section{Conclusions}

A purposely established UV-LIGA process for the fabrication of moderate aspect ratio metal waveguides for sub- $\mathrm{THz}$ frequencies has been described. The use of multiple layers of SU-8 to achieve the required thickness of the metal structure, with high planarity, has been demonstrated as an effective approach. The improvement in the planarization of the SU-8 surface at the increase of the number of layers is fundamental to have a high quality flat metal surface as ground planes or assembly surfaces. The fabrication of the metal DCW demonstrates the excellent quality obtained by the presented process that will enable the affordable fabrication of sub-THz devices for a wide range of applications.

\section{Acknowledgement}

The authors would like to thank Dr. Andrew Marshall of Physics Department and Dr. Farid Aiouache for their support and advice in the course of this project. This work was supported by the UK EPSRC EP/L026597/1 grant.

\section{References}

[1] Booske J H, Dobbs R J , Joye C D, Kory C L, Neil G R, Park G S, Park J H and Temkin R J 2011 "Vacuum electronic high power terahertz sources," IEEE Trans. Terahertz Sci. Technol., 1, 54-75

[2] Joye C D, Calame J P, Garven M and Levush B 2010, "UV-LIGA microfabrication of $220 \mathrm{GHz}$ sheet beam amplifier gratings with SU-8 photoresists," J. Micromech. Microeng., 20, 125016

[3] Joye C D, Calame J P, Nguyen K T and Garven M 2011 "Microfabrication of fine electron beam tunnels using UVLIGA and embedded polymer monofilaments for vacuum electron devices J. Micromech. Microeng. 22015010

[4] Gamzina D, Himes L, Barchfeld R, Popovic B, Pan P, Letizia R, Mineo M, Feng J, Paoloni C and Luhmann Jr. N C 2016 Nanoscale Surface Roughness Effects on THz Vacuum Electron Device Performance IEEE Trans. Nanotechnol., 15, 85-93

[5] Paoloni C, Di Carlo A, Bouamrane F, Bouvet T, Durand A J, Kotiranta M, Krozer V, Megtert S, Mineo M and Zhurbenko V 2013 Design and Realization Aspects of 1THz Cascade Backward Wave Amplifier Based on Double Corrugated Waveguide IEEE Trans. on Electron Devices, $60,1236-1243$

[6] Shin Y M, Barnett L R, Gamzina D, Luhmann Jr. N C, Field M and Borwick R 2009 Terahertz vacuum electronic circuits fabricated by UV lithographic molding and deep reactive ion etching Appl. Phys. Lett., 95, 181505

[7] Xie F, Ding G, Zhao X, and Cheng P 2015 Design, fabrication and measurement of a novel $140 \mathrm{GHz}$ folded waveguide based on SU-8 UV-LIGA technology $J$. Micromech. Microeng., 25, 085010

[8] Shin Y, Park G, Scheitrum G P and Arfin B 2003 Novel Coupled-Cavity TWT Structure Using Two-Step LIGA Fabrication IEEE Trans. Plasma Sci., 31, 1317-1324

[9] Tucek J C, Basten M A, Gallagher D A and Kreischer K E 2016 Northrop Grumman Operation of a Compact 1.03 THz Power Amplifier"" Proc. 17th IEEE Int. Vacuum Electronics Conf., (Monterey, CA) (New York) 37-38

[10] Siegel D H 2002 Terahertz technology IEEE Trans. Microw. Theory Techn. 50, 910-928

[11] Tonouchi M 2007 Cutting-edge terahertz technology Nature Photon., 1, 97-105

[12] Paoloni C, Gamzina D, Himes L, Popovic B, Barchfeld R, Yue L, Zheng Y, Tang X, Tang Y, Pan P, Li H, Letizia R, Mineo M, Feng, J and Luhmann Jr., NC 2016 THz Backward-Wave Oscillators for Plasma Diagnostic in Nuclear Fusion, IEEE Trans. On Plasma Sci., 44, 369-37

[13] Malekabadi A, Charlebois S A, Deslandes D and Boone F 2014 High-Resistivity Silicon Dielectric Ribbon Waveguide for Single-Mode Low-Loss Propagation at F/GBands," IEEE Trans. Terahertz Sci. Technol., 4, p. 7

[14] Chen K, Ayón A A, Member S, Zhang X and Spearing S M 2002 Effect of Process Parameters on the Surface Morphology and Mechanical Performance of Silicon Structures After Deep Reactive Ion Etching (DRIE) Journal of Microelectromech. Sys., 11, 264-275

[15] Miller K, Li M X, Walsh K M, and X. a Fu, 2013 The effects of DRIE operational parameters on vertically aligned micropillar arrays," J. Micromech. Microeng., 23, 035039

[16] del Campo A and Greiner C 2007 SU-8: a photoresist for high-aspect-ratio and 3D photolithography J. Micromech. Microeng. 17 R81-95

[17] Shin Y M, Gamzina D, Barnett L R, Yaghmaie F, Baig A, Luhmann Jr. N C 2010 UV Lithography and Molding Fabrication of Ultrathick Micrometallic Structures Using a KMPR Photoresist Journal of Microelectromech. Sys., 19, 683

[18] Mineo M and Paoloni C 2010 Double-corrugated rectangular waveguide slow-wave structure for terahertz vacuum devices IEEE Trans. Electron Dev., 57, 3169-3175

[19] Mineo M and Paoloni C 2010 Corrugated Rectangular Waveguide Tunable Backward Wave Oscillator for $\mathrm{THz}$ Applications IEEE Trans. on Electron Dev., 57, 1481-1484

[20] Microchem, "SU-8 2000 Permanent Epoxy Negative Photoresist," 2000

[21] Lin C-H, Lee G-B, Chang B-W and Chang G-L 2002 A newfabrication process for ultra-thick microfluidic microstructures utilizing SU-8 photoresist J. Micromech. Microeng. 12 590-7

[22] Hong G, Holmes A S and Heaton M E 2004 SU8 resist plasma etching and its optimisation Microsyst. Technol., 10, 357-359

[23] Engelke R, Mathuni J, Ahrens G, Gruetzner G, Bednarzik M, Schondelmaier D and Loechel B 2008 Investigations of SU-8 removal from metallic high aspect ratio microstructures with a novel plasma technique $J$. Microsyst. Technol. 14 1607-12 